\title{
On the optical and thermal properties of in situ/ex situ reduced Ag NP's/PVA composites and its role as a simple SPR-based protein sensor
}

\author{
A. Nimrodh Ananth · S. Umapathy • \\ J. Sophia $\cdot$ T. Mathavan $\cdot$ D. Mangalaraj
}

Received: 28 February 2011/Accepted: 18 April 2011/Published online: 12 July 2011

(C) The Author(s) 2011. This article is published with open access at Springerlink.com

\begin{abstract}
Silver/polyvinyl alcohol (Ag-PVA) nanocomposite films have been prepared via in situ generation of silver nanoparticles (Ag NPs) by the respective metallic salts and dispersion of preformed Ag NPs (ex situ synthesis) inside polyvinyl alcohol (PVA) and its effect of sensing towards a model protein (bovine serum albuminBSA) was investigated. The influence of Ag NPs, irrespective of their reduction methodology on the optical and the thermal properties of the PVA, had been investigated using UV-Vis spectrophotometer and differential scanning calorimetry. The absorption peak around $400 \mathrm{~nm}$ indicates the surface plasmon resonance response of Ag NPs. The interaction of the dispersed and preformed Ag NPs with the PVA chains is confirmed by the corresponding vibrational signatures of the PVA through Fourier transform infrared spectroscopy (FTIR). The changes in the glass transition and melting temperatures $\left(T_{\mathrm{g}}\right.$ and $\left.T_{\mathrm{m}}\right)$ of the pure PVA upon the presence of Ag NPs are reported using differential scanning calorimeter (DSC). The sizes of the synthesized Ag NPs are found to be in the range of $200 \pm 10 \mathrm{~nm}$ for in
\end{abstract}

Electronic supplementary material The online version of this article (doi:10.1007/s13204-011-0010-7) contains supplementary material, which is available to authorized users.

A. Nimrodh Ananth $(\bowtie) \cdot$ S. Umapathy $\cdot$ J. Sophia

Polymer Laboratory, School of Physics, Madurai Kamaraj

University, Madurai 625 021, India

e-mail: a.nimrodh@gmail.com

\section{T. Mathavan}

Department of Physics, NMSSVN College,

Nagamalai, Madurai 625 019, India

D. Mangalaraj

Department of Nanoscience and Technology,

Bharathiar University, Coimbatore 641 046, India situ reduction of silver nitrate $\left(\mathrm{AgNO}_{3}\right)$ and $100 \pm 10 \mathrm{~nm}$ for the external addition of preformed Ag NPs by sodium borohydride $\left(\mathrm{NaBH}_{4}\right)$ reduction using scanning electron microscopy (SEM).

Keywords Surface plasmon resonance . In situ generation $\cdot$ Ag-PVA $\cdot$ BSA

\section{Introduction}

Metal nanoparticles incorporated polymers attracted great attention because of the widened application scope offered by these hybrid materials (Nesher et al 2008; Wang et al. 2002; Clemenson et al. 2006; Akamatsu et al. 2000; Zeng et al. 2002). Polymers are considered as good host materials for metal nano colloids by providing a protective coating layer over the highly active particles. At the same time, these embedded nanoparticles inside the polymer matrix will also affect the properties of the host itself (Akamatsu et al. 2000; Zeng et al. 2002; Hussain et al. 2003; Zavyalov et al. 2002; Lee et al. 2008). Particularly, polymer-metal hybrids such as polymer-silver composites are promising functional materials in fields such as optical, magnetic, electronic, and antimicrobial properties (Zhong et al. 2003; Hopkins et al. 2005; Clemenson et al. 2008; Temgire and Joshi 2004; Zheng et al. 2000). Silver nanoparticles have received considerable attention due to their attractive physical and chemical properties and are extensively investigated in the areas of bio-sensing (Anker et al 2008; McDonagh et al 2008). Silver nanoparticles protected by polymers such as PVA, PVP, PMMA are extensively reported (Zhou et al. 1999; Chou and Ren 2000; Khanna et al. 2004; Zhang et al. 1996; Monti et al. 2004). PVA could be considered as a good host material for metal due to its excellent thermo stability 
and chemical resistance (Fusell et al. 2005; Porel et al. 2005). PVA can effectively protect the nanoparticles from aggregation (Khanna et al. 2005). The effect induced by the embedment of Ag NPs, in situ and ex situ, on the polymer host are very sparingly reported in the literature (Porel et al. 2005; Khanna et al. 2005; Gautam and Ram 2009; Devi et al. 2002; Karthikeyan 2005; Mbhele et al. 2003; Filippo et al. 2009; Yu et al. 2007).

The major objective of this study was to synthesize, stabilize silver nano particles inside the PVA host with and without employing conventional reducing agents and to study the optical and thermal property variation of the supporting polymer and also to investigate the effect of embedded silver nano system as a simple surface plasmon resonance-based sensor for sensing of a model protein bovine serum albumin (BSA). The presence of silver nanoparticles embedded inside the polymer has been confirmed by the surface plasmon resonance response. The morphology of the formed nanoparticles has been found through scanning electron microscopy. The response of optical band gap, glass transition and melting temperatures on PVA along with Ag NPs embedment is also investigated. The effect of sensing of BSA by PVA-encapsulated silver nanoparticles is investigated through the surface plasmon resonance response of silver nanoparticles upon the addition of BSA with desired concentrations on shortterm duration.

\section{Experimental}

Partially hydrolyzed PVA (S.D. Fine Chem. Ltd., AR) of average molecular weight of 1,25,000 Da, has been dissolved in the mixture of propane-2-ol and double deionized water (1:1), in order to have 2\% (w/v) PVA aqueous solution, with a mild stirring and the aqueous solution has been left overnight on the stirrer. Bovine serum albumin (Sigma - AR) of molecular weight $\sim 6,500 \mathrm{Da}$ had been utilized as a model protein for the purpose of sensing. All glassware has been extensively washed with aqua regia and rinsed with double deionized water several times and oven dried until use.

PVA-encapsulated silver nanoparticle had been achieved via two distinct approaches: (1) synthesis of $\mathrm{Ag}$ NPs by sodium borohydride and stabilization of Ag NPs with PVA, (2) simultaneous synthesis and stabilization of Ag NPs using PVA.

Synthesis of Ag NPs by sodium borohydride and stabilization with PVA

Reduction of silver nitrate (Loba Chemie, Mumbai, India99\%, AR) to its own metal counterpart had been assisted with sodium borohydride (Loba Chemie, Mumbai, India97\%-AR) with standard protocol (Mulfinger et al. 2007). The schematic representation of ex situ reduced silver nitrate/PVA is shown in Fig. 2a. Briefly, $0.01 \mathrm{M}$ silver nitrate is added drop by drop to $0.02 \mathrm{M}$ freshly prepared ice cold sodium borohydride with stirring. The visible color changes from colorless to dark yellow after the addition of silver nitrate indicating the formation of silver nano colloids. The stirring is terminated after complete addition of $\mathrm{AgNO}_{3}$ to prevent the aggregation. The formed Ag NPs are injected into the aqueous PVA solution with gentle stirring and is left for about $30 \mathrm{~min}$. The change of dark yellow solution to mild yellow indicates the encapsulation (Diez I et al. 2009). The resulting colloidal dispersion was casted onto a pre-cleaned Petri dish to yield micron sized films which had been utilized for investigating the effect of silver nanoparticles in the optical and thermal variations of PVA. These films can also be re-dispersed in the utilized solvent to form the native colloidal dispersion (Fig. 1) of PVAencapsulated silver nanoparticles and this colloidal system had been studied for its sensing ability towards BSA.

Simultaneous synthesis and stabilization of Ag NPs using PVA

The schematic representation of in situ reduced silver nitrate/PVA is shown in Fig. 2b. In this procedure, $0.01 \mathrm{M}$ of freshly prepared silver nitrate $(0.01 \mathrm{M})$ is carefully injected into aqueous PVA solution in the ratio of 1:5 with gentle stirring and is left for $30 \mathrm{~min}$. This homogenized solution (PVA $+\mathrm{AgNO}_{3}$ ) is casted onto a clean Petri dish. Then it is subjected to $50^{\circ} \mathrm{C}$ controlled environment for $24 \mathrm{~h}$ after which the resulted colored films are peeled off from the support for further characterization. PVA by itself, without any assistance of other agents, is optically stable (see supporting information).

The influence of the methodology of silver nanoparticle incorporation has also been analyzed, the capability of the polymer host, PVA to keep the metal precursor stable for a long time until any external initiation (see supporting information) for reduction provides the experimenter to decide his own final product according to his needs which is an added advantage of the system when compared with that of the ex situ methodology which was also prone to aggregation with respect to several situations which are completely absent in the in situ process.

\section{Characterization}

Shimadzu UV-2450 spectrophotometer with a resolution of $0.1 \mathrm{~nm}$ is used to identify the formation of silver nanoparticles and to investigate the influence of the embedded 
Fig. 1 Illustration of the PVAencapsulated silver nanoparticles in colloidal nature and in solid staterepresentation of flexibility in acquiring the samples in desired modes
Fig. 2 a, b Schematic representation of ex situ/in situ reduced $\mathrm{Ag} / \mathrm{PVA}$ composite

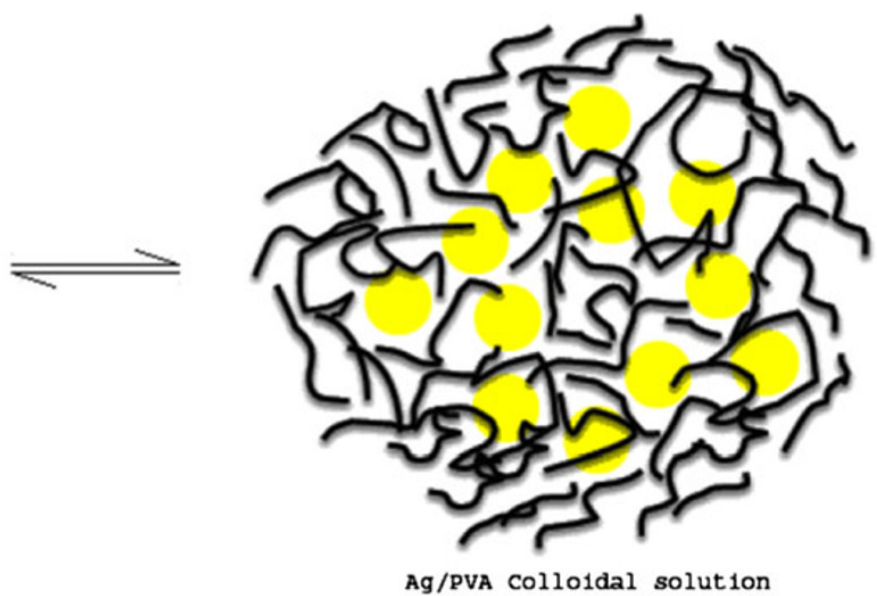

s1lver Nanopart1cle (sensing element)

PVA Chain

Ex-situ reduced Metal Salts/Polymer

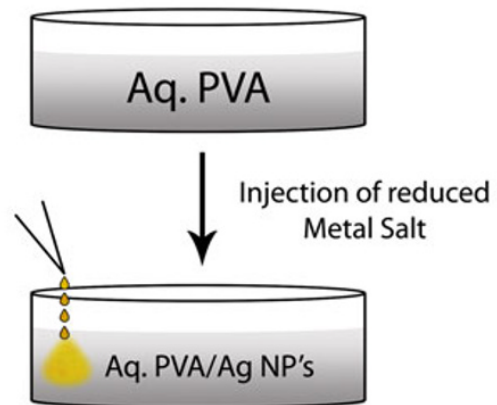

Casted in Petridish \& allowed to dry for 24 hours at room temperature

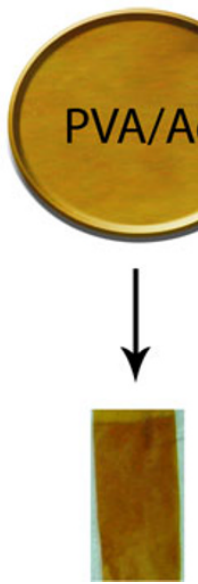

Free standing film Ag Embedded PVA
In-situ reduced Metal Salts/Polymer
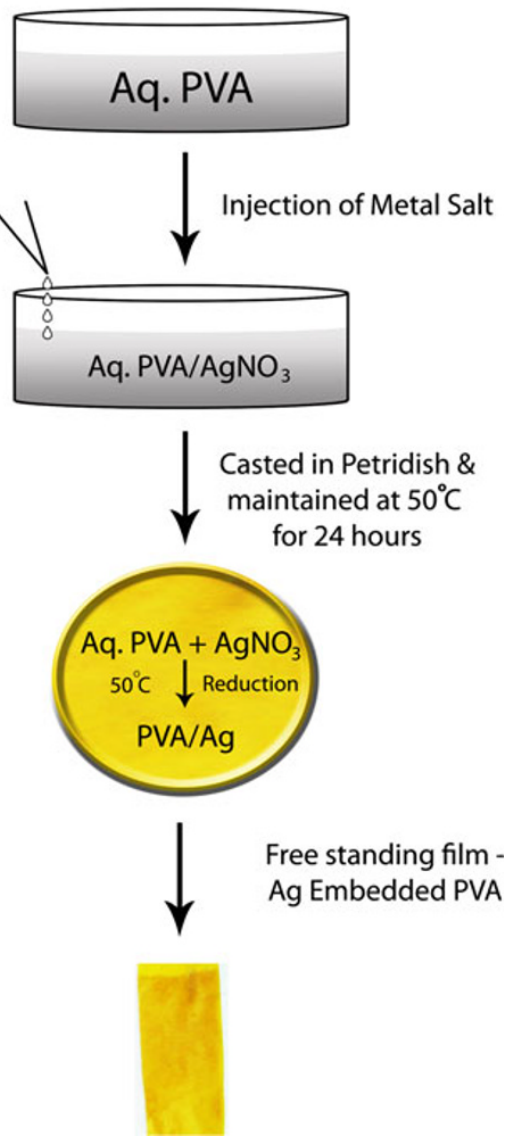
Ag Embedded PVA silver nanoparticles, irrespective of their synthesis methodology, on the optical band gap of the pure PVA and also as a tool for analyzing the colloidal sample as a simple
SPR-based optical sensor. Shimadzu FTIR-8400S spectrophotometer is employed to investigate the formation of Ag-PVA composites. Jeol version 1.0 scanning electron 
microscopy (SEM) is used for observing the morphology of silver nanoparticles inside PVA. Thermal analyses are performed by differential scanning calorimetry (DSC Q20 V24.2) at a heating rate of $10 \mathrm{~K} / \mathrm{min}$ in nitrogen atmosphere.

\section{Results and discussion}

The absorption peaks at 400 and $429 \mathrm{~nm}$ in Fig. 3 show the characteristic surface plasmon peak dedicated to silver nanoparticles (Khanna et al. 2005; Karthikeyan 2005). The graph ' $a$ ' in the Fig. 3 corresponds to the ex situ synthesis of silver nanoparticles stabilized with polyvinyl alcohol, whereas the graph ' $b$ ' corresponds to the in situ generation of silver nanoparticle in PVA. In the second case, PVA serves as both stabilizing and reducing agent (see supporting information), from the fact that the vinyl polymers with high density of polar groups facilitate the reduction process (Rozenberg and Tenne 2008).

The full width half maximum (FWHM) of the respective absorption patterns of ex situ/in situ generated Ag NPs are found to be 64.30 and $99.13 \mathrm{~nm}$. This reveals that the size distribution of the particles is broader (Ershov et al. 1993) for in situ synthesized silver nanoparticles when compared with the addition of preformed nanoparticles. This is also supported by the observed red shift for in situ synthesized silver nanoparticles. Also, the difference in the peak intensity is due to the concentration of the silver inside the host which can also be observed through the colors of the films. These colors also indicate the expected surface plasmon resonance and found shift in the position too (Mock et al. 2008). The increase in the particle size may be due to the constant temperature and the time constrains forced to facilitate the reduction. The increase in the temperature with reduced time or prolonged exposure time of the casted films in the moderate temperature compartment

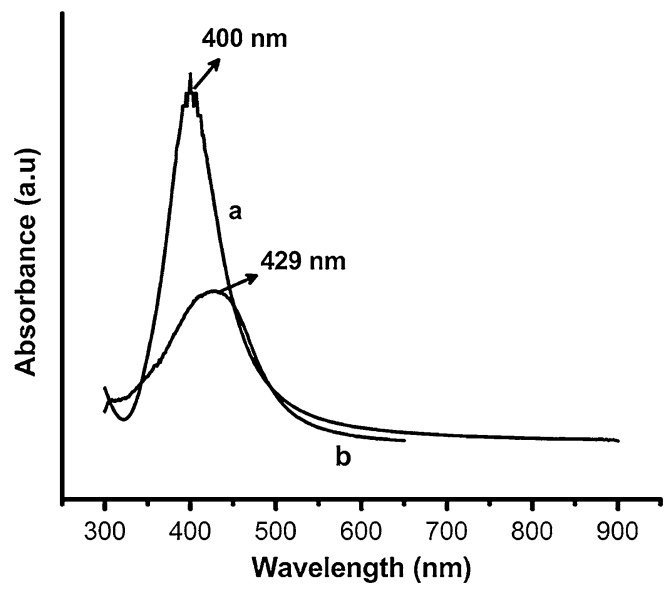

Fig. 3 Absorbance of $a \mathrm{Ag}$ (ex situ)/PVA, $b \mathrm{Ag}$ (in situ)/PVA can induce considerable effects in the size and distribution of the embedded silver nanoparticles (Khanna et al. 2009). The incorporation of the silver nanoparticles, irrespective of their methodology of synthesis, also affects the band gap of the involved polymer system. This also confirms the presence of the inorganic fillers inside the host. The observed direct and indirect band gaps of pure PVA are found to be 5.23 and $4.89 \mathrm{eV}$ as seen from Fig. 4a (A, B). These are consistent with the literature (Devi et al. 2002; Mbhele et al. 2003). The graph between $(\alpha \mathrm{h} v)^{n}$ verses $\mathrm{h} v$ (photon energy) for $n=2$ (direct) and 0.5 (indirect) is plotted. For $\mathrm{Ag}$ (ex situ)/PVA films, the direct and indirect optical band gaps are found to be 5.12 and $4.51 \mathrm{eV}$ from Fig. 4c (A, B), respectively.

In the case of silver nanoparticles prepared and embedded by the polymer, the direct and indirect optical band gaps are found to be 4.59 and $3.99 \mathrm{eV}$ as seen from Figs. $4 \mathrm{~b}$ and $5 \mathrm{~b}$, respectively. The direct and indirect optical band gaps of the samples are shown in the Table 1 .

The values for the pure PVA agree well with literature (Devi et al. 2002; Mbhele et al. 2003). As shown in the Table 1, the optical band gaps decreases after the addition of silver nanoparticles can be explained on the basis that the incorporated silver nanoparticle acts as a donor to the polymer host thereby forming charge-transfer complexes. The nature of these interactions is also supported by the FTIR investigations. Also, we believe that there may be an inducement from particle size, nature and the environment in altering the optical band gaps of the Ag (in situ)/PVA films in reference with pure PVA. Since the reduction of the silver nitrate to silver can affect the formation of the films.

The incorporated and generated silver nanoparticles inside PVA will considerably change the properties of the polymer involved. The composite formation can also be interpreted through the alignments in the finger print regions of pure PVA after the incorporations of the ex situ/ in situ employed silver nanoparticles in the polymer host. These films show a very good optical clarity. Interactions of silver nanoparticles with the pure polymer are searched through the recorded transmittance pattern. Figure 5 shows the response of pure and Ag-embedded PVA in the finger print region. The inset of the Fig. 5 indicated with arrows shows the silver nanoparticle interacted region of PVA. The increase in the transmittance of the silver-embedded PVA films was observed with the prominent increase in the corresponding vibrational frequency dedicated for in-plane $\mathrm{O}-\mathrm{H}$ vibrations with $\mathrm{C}-\mathrm{H}$ wagging vibrations at 1,420 and $1,379 \mathrm{~cm}^{-1}$. This indicates the interactions of embedded silver inside the PVA matrix, in particular with the $\mathrm{O}-\mathrm{H}$ group of PVA. The broad peak covering $550-750 \mathrm{~cm}^{-1}$ corresponds to out-of-plane O-H vibration (Karthikeyan 2005; Mbhele et al. 2003).

The formation of Ag-PVA composites are also supported by the SEM micrographs. The embedded silver 

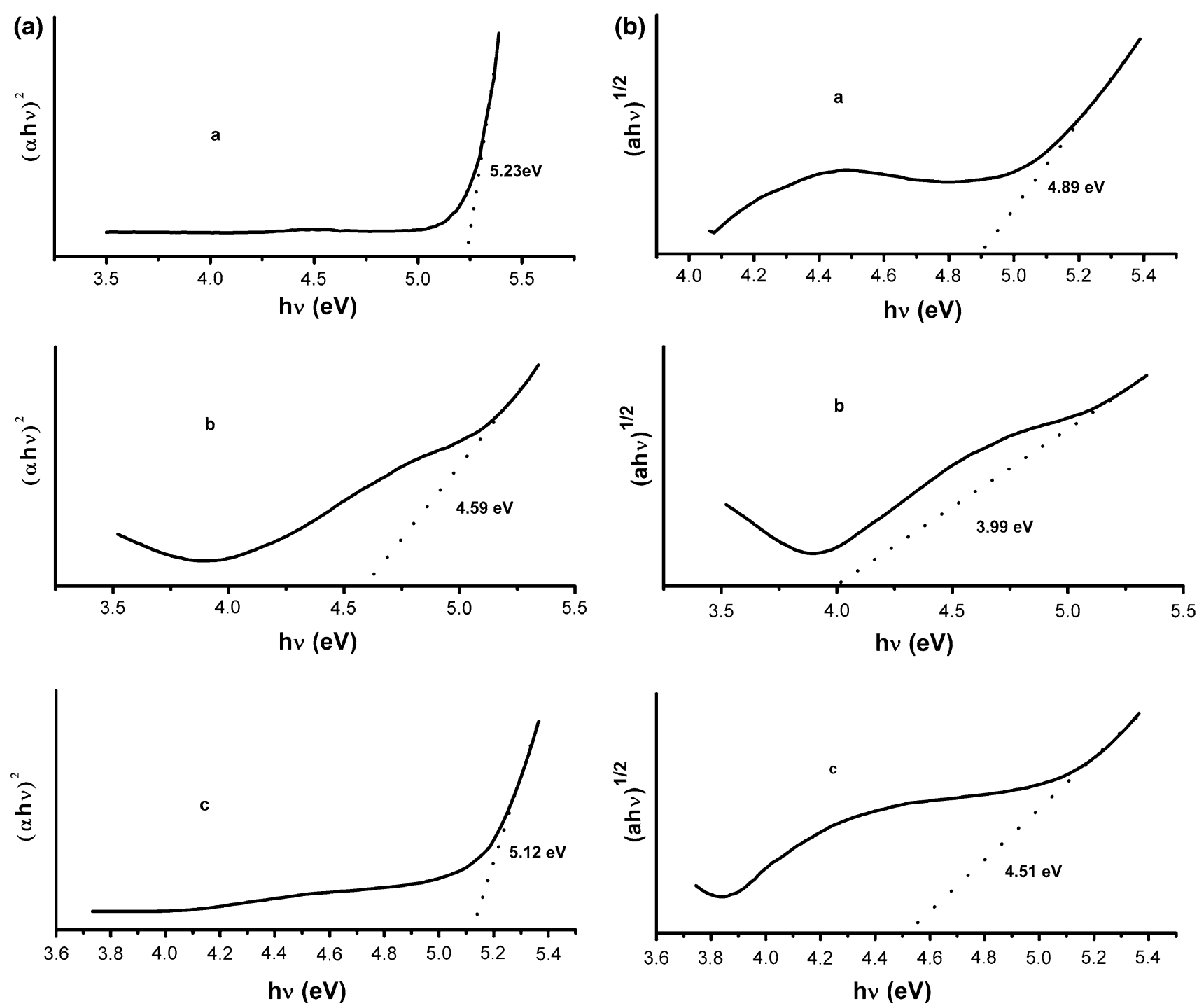

Fig. 4 A, B Plot to determine the direct band gap and indirect band gap of $a$ Pure PVA, $b$ Ag (in situ)/PVA, $c$ Ag (ex situ)/PVA

nanoparticles, both in situ generated and ex situ, in PVA. These are analyzed through SEM for their size and morphology. The Ag (ex-situ)-PVA and Ag (in situ)-PVA films are re-dispersed in the referred solvent for SEM measurements after a month from synthesis. The recorded SEM micrographs for Ag (ex-situ)-PVA are prone to aggregation which may be the result of aggregation before the transfer of silver nanoparticles to the aqueous PVA solution. We have indicated the presence of small silver structures through arrows as shown in Fig. 6b. The size distribution of these clusters is found to be in the range of $100 \pm 10 \mathrm{~nm}$. Similarly, the observed size distribution for the in situ reduced and embedded silver nanoparticles in PVA is $200 \pm 10 \mathrm{~nm}$, but the uniformity and square shape of in situ reduced and embedded silver nanoparticles are unique when compared with that of the injected performed silver nanoparticles inside the host (Fig. 7). This may be
Table 1 Optical band gaps for pure PVA and both Ag-embedded PVA system

\begin{tabular}{lll}
\hline Sample & \multicolumn{2}{l}{ Optical band gap } \\
\cline { 2 - 3 } & Direct $(\mathrm{eV})$ & Indirect $(\mathrm{eV})$ \\
\hline Pure PVA & 5.20 & 4.89 \\
Ag (ex situ)/PVA & 5.12 & 4.60 \\
Ag (in situ)/PVA & 4.59 & 4.00 \\
\hline
\end{tabular}

due to the constant temperature environment which facilitates the reduction process of the $\mathrm{AgNO}_{3}$-PVA homogenous solution. Any changes in the reaction conditions or the environments will affect the resulting product. This may be the result of utilizing the PVA as reducing as well as stabilizing agent. The particle size and its shape can also be well tuned as per our own preference like increasing the 


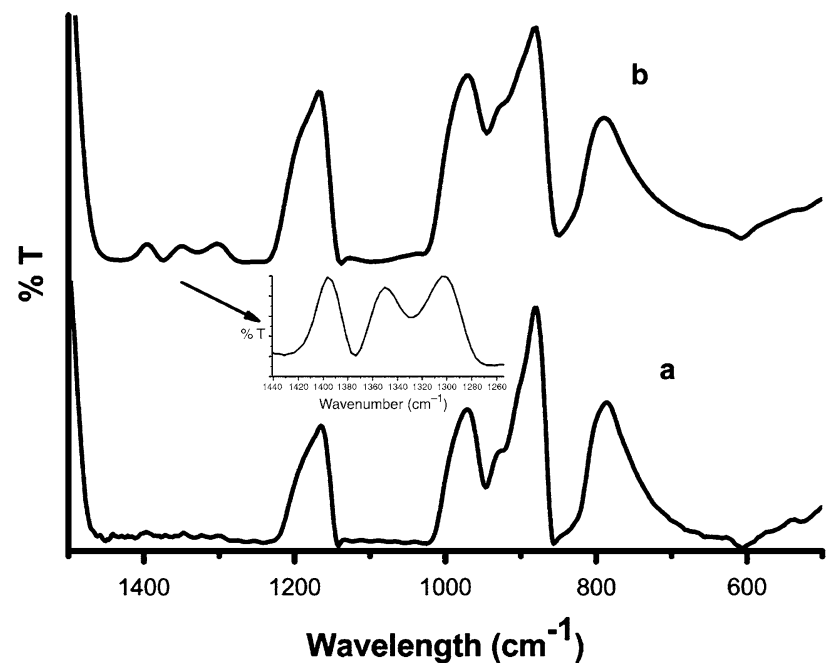

Fig. 5 FTIR spectra of $a$ pure PVA, $b$ Ag-embedded PVA
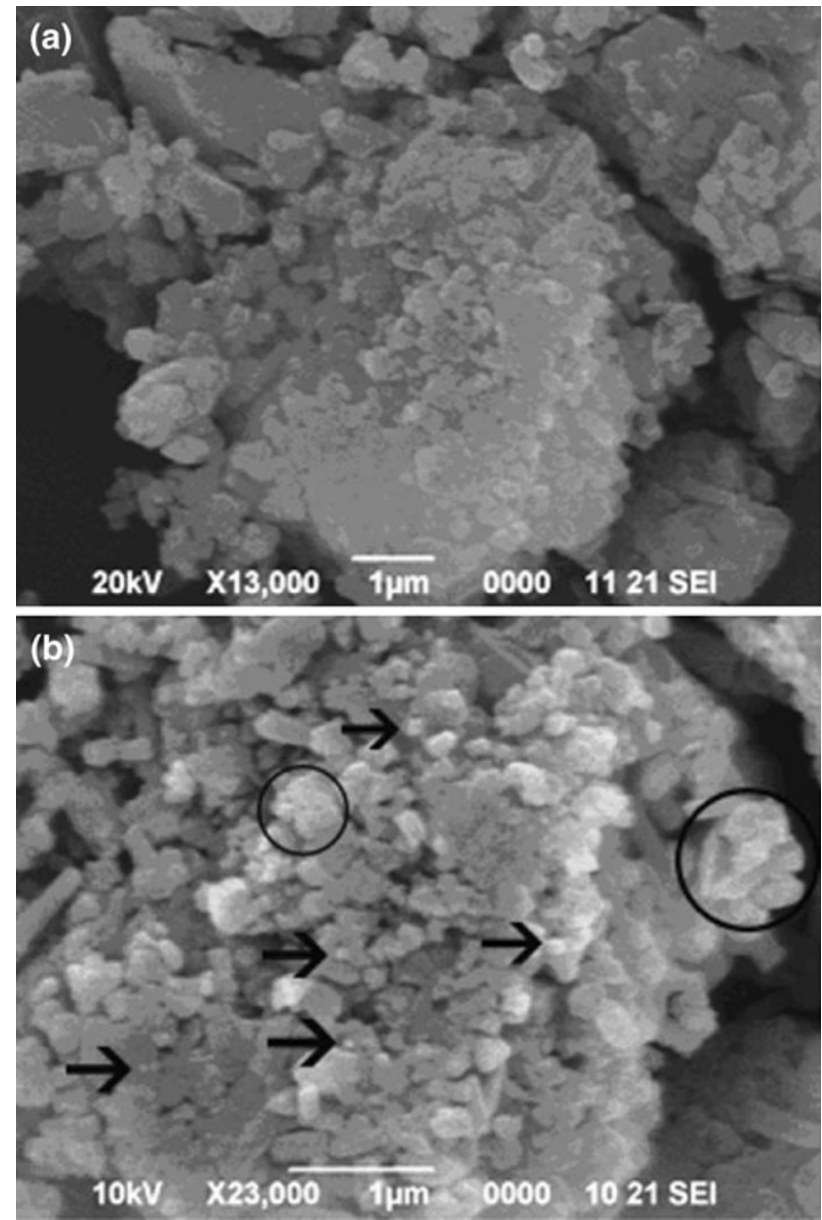

Fig. 6 a, b SEM image of Ag (ex situ)/PVA sample, for different magnification

temperature environment of the $\mathrm{PVA}-\mathrm{AgNO}_{3}$ homogenous casted solutions which, here in this case, is believed to induce PVA in the process of reduction.
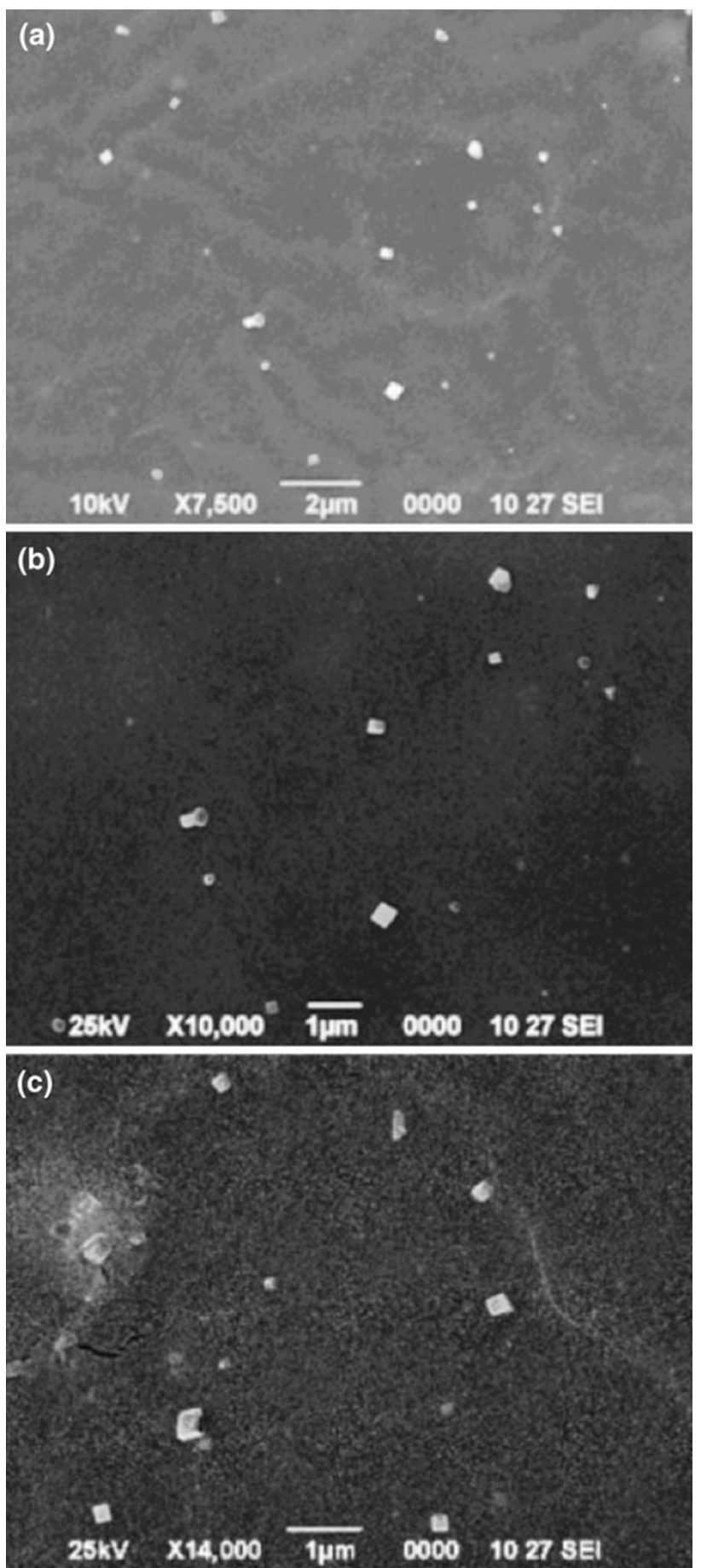

Fig. 7 a, b, c SEM image of Ag (in situ)/PVA samples, for different magnification

The difference in the particle shape, its distribution between the two different modes of reduction and embedment are very well supported by the red shift in the peak position in the second case (in situ reduction and embedment), as seen from Fig. 3. This is also confirmed by the increase in the FWHM values derived from its 

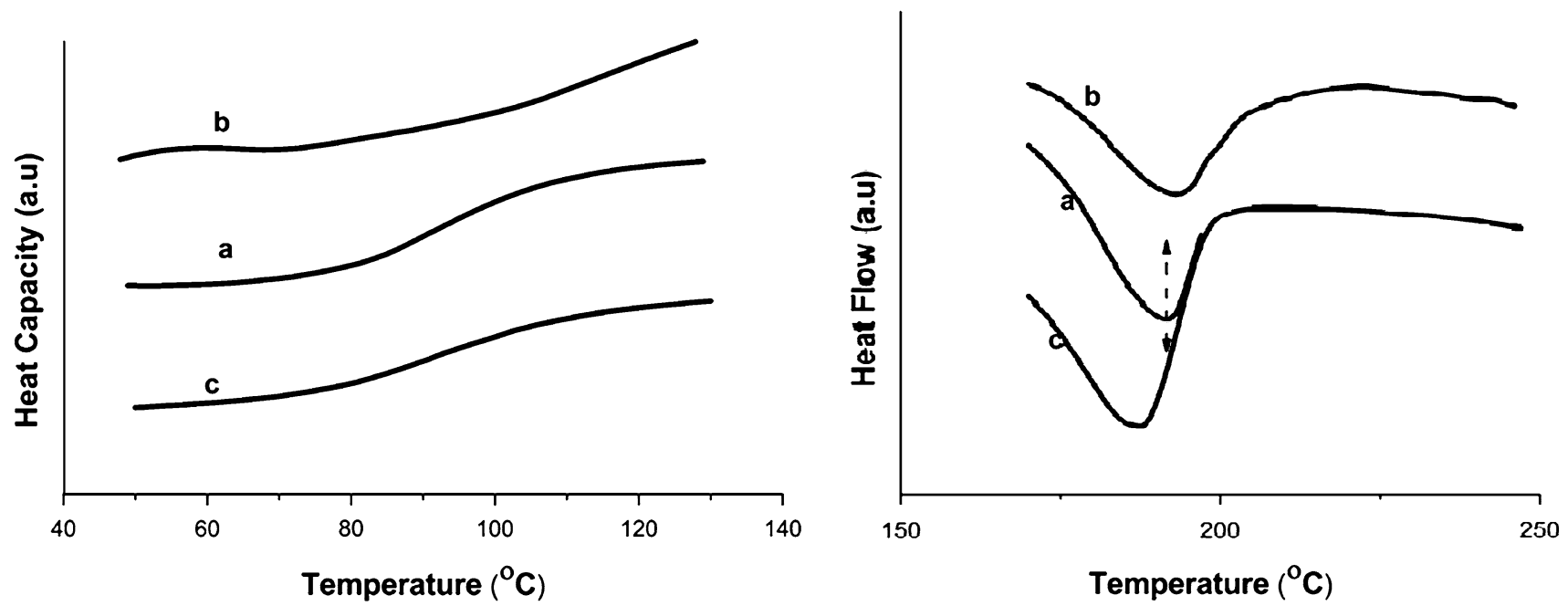

Fig. 8 A, B Glass transition temperature and melting points of $a$ pure PVA, $b$ Ag (in situ)/PVA, $c$ Ag (ex situ)/PVA

Table 2 Melting peak $\left(T_{\mathrm{m}}\right)$ and glass transition temperature $\left(T_{\mathrm{g}}\right)$ of the samples

\begin{tabular}{lll}
\hline Sample & $T_{\mathrm{m}}\left({ }^{\circ} \mathrm{C}\right)$ & $T_{\mathrm{g}}\left({ }^{\circ} \mathrm{C}\right)$ \\
\hline Pure PVA & 191.65 & 85.78 \\
Ag (ex situ)/PVA & 187.43 & 97.46 \\
Ag (in situ)/PVA & 193.40 & 80.64 \\
\hline
\end{tabular}

absorption spectra in comparison with the embedment of preformed silver nanoparticles. The formation of the composite in the first case and the formation of silver nanoparticles in the second case, as shown in Figs. 6 and 7 are hence confirmed by the SEM images.

The DSC heating curves of the pure PVA and Ag-PVA nanocomposites are divided into two temperature regions (from 50 to $130^{\circ} \mathrm{C}$, Fig. $8 \mathrm{~A}$ and from 130 to $220^{\circ} \mathrm{C}$, Fig. $8 \mathrm{~B}$ ). Table 2 gives the glass transition temperature $\left(T_{\mathrm{g}}\right)$ and melting point $\left(T_{\mathrm{m}}\right)$. The increase in the $T_{\mathrm{g}}$, as shown in Fig. 8A after the incorporation of preformed silver nanoparticles into the host can be explained on the basis of interactions of the injected silver nanoparticles with the chains of PVA resulting in the reduction in the chain mobility. This explanation is inconsistent with the literature (Mbhele et al. 2003).

But it is not the case in Ag (in situ)/PVA film which indicates that $T_{\mathrm{g}}$ of the PVA not only depends on the concentration of the incorporated inorganic fillers but also it should have a considerable impact from the nature of the fillers (Mbhele et al. 2003).

The graphs a, b and c shown in Fig. 8B correspond to the melting peak of pure PVA, Ag-embedded PVA, both $b$ and $\mathrm{c}$ which correspond to forced injection of the preformed Ag colloids and in situ reduction/stabilization of silver by the host itself, respectively. The observed broadening and increase in the melting temperature of $\mathrm{Ag}$ (in situ)/PVA sample in comparison with the pure PVA peak, as shown in Fig. 8B can be explained on the basis of the reduced mobility of the PVA chains resulted from the interactions of $\mathrm{Ag}$ with the polymer (Mbhele et al. 2003). These patterns are also supported by the variations in the intensity profiles observed from FTIR data. It may also be believed that particle size and nature of these fillers will play a role in tuning the thermal properties of the polymers.

\section{Ag/PVA colloidal system: a simple SPR-based optical sensor}

The study had been extended to investigate the potentiality of the PVA-encapsulated Ag NPs towards a sensing of a biological macromolecule (model protein-BSA). BSA had been chosen as a model protein because of its wide range of physiological functions and also due to its water soluble nature which is very important for interaction studies (Valanciunaite et al. 2006; Hansen 1981).

Two different concentrations of BSA $(0.1$ and $1 \mathrm{mM})$ were taken and injected into PVA-encapsulated Ag NPs colloidal solution in order to investigate response, in a timely fashion, of the colloidal system upon protein incorporation. The time-dependent responses were investigated through UV-Vis spectrophotometer with 6 min time interval up to $60 \mathrm{~min}$.

It is clear that upon the incorporation of BSA at the desired concentrations, there is a decrease in the absorption pattern with a notable shift in the wavelength regime, towards red ( $\Delta \lambda_{\mathrm{SPR}} \approx 3 \mathrm{~nm}$, for both concentrations). The rate of change in the intensity of the SPR profiles upon the addition of BSA, certain concentration follows a power law rate of -0.02 and -0.05 for increasing concentration with correlation of 0.992 and 0.996 for both 0.1 and $1.0 \mathrm{mM}$ 


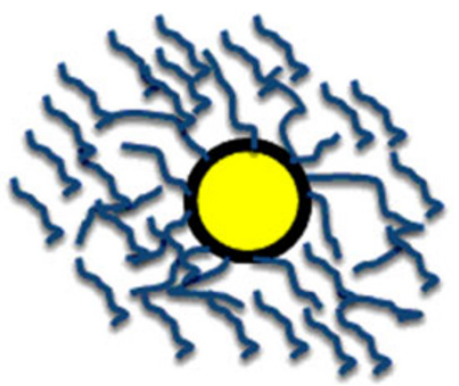

s1lver Nanopart1cles (sens1ng element)

PVA Cha1n

BSA

Fig. 9 Illustration of PVA-encapsulated silver nanoparticles in sensing BSA

concentrations of BSA. Neat silver colloidal solution, due to its instability, shows the signature of coagulation when utilized for the purpose of sensing (see supporting Information).

It is also noted that at larger time scales, the variations in the intensity profile almost vanishes which is due to the fact

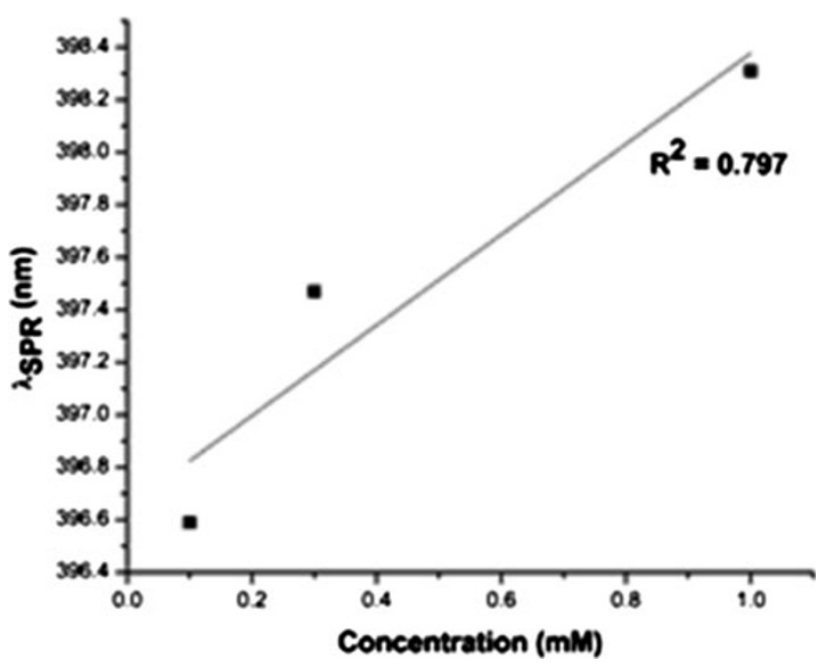

Fig. 11 SPR shift of PVA-encapsulated silver nanoparticle with respect to concentration of BSA

that the adsorbed protein will form a layer on the sensing system thereby preventing excess BSA fragments from the vicinity of the sensing element (Fig. 9) which is also supported by the plasmon shift towards red wavelength. The deviation of SPR position with respect to concentration of BSA, as in Fig. 11, indicates the sensitivity of the system towards the analyte concentration. PVA-encapsulated
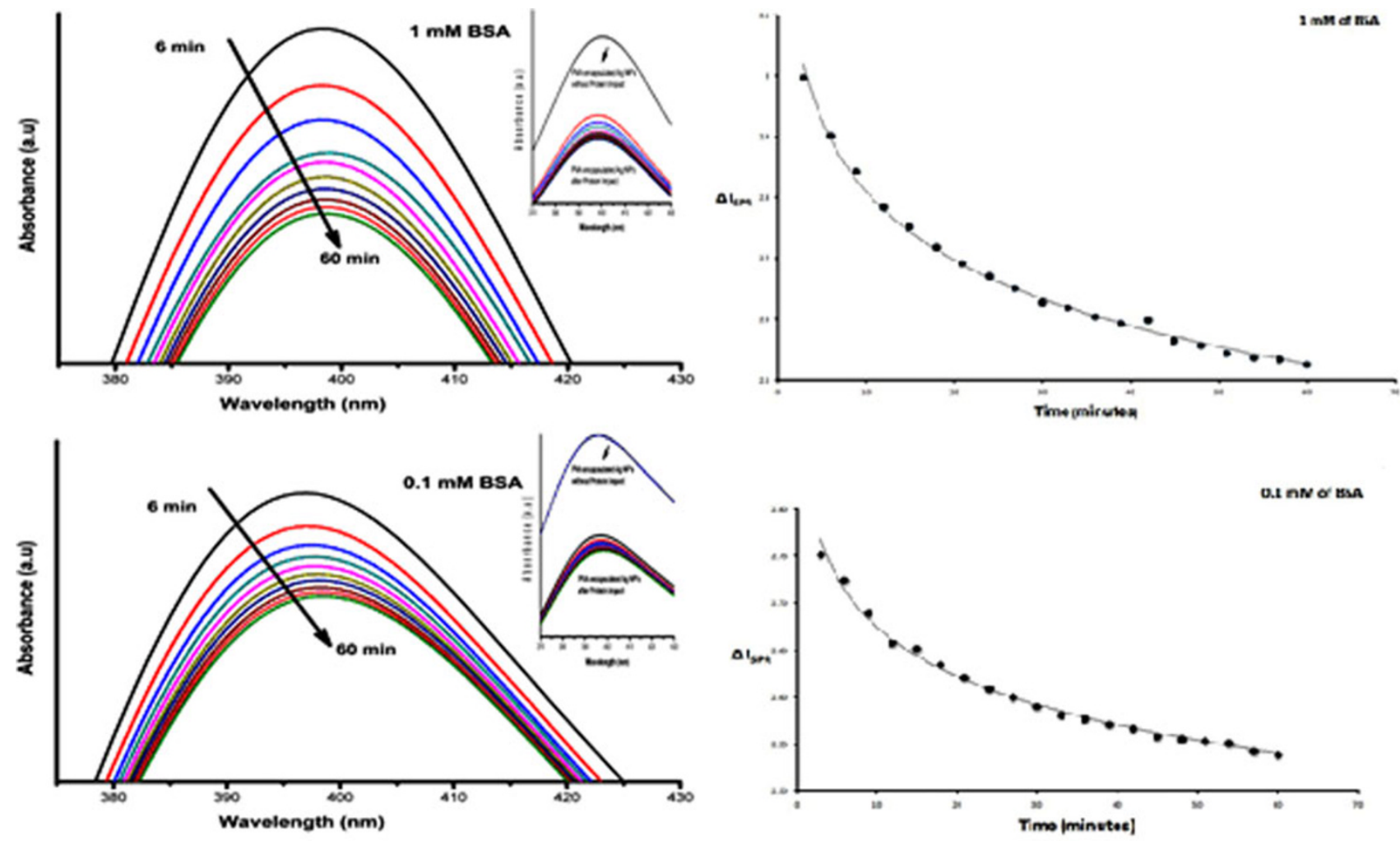

Fig. 10 Response of PVA-encapsulated silver nanoparticles upon BSA incorporation and its respective intensity profiles 
silver nanoparticles can also act as a simple SPR-based sensing element for biologically important macromolecules (Fig. 10).

\section{Conclusion}

In situ and ex situ reduction of silver nitrate to silver nanoparticles are performed with simultaneous stabilization and reduction in PVA host for first case (in situ) and for stabilization alone in the second system (ex situ). The presence of surface plasmon band at 400 and $429 \mathrm{~nm}$ indicated the formation of silver nanoparticles in the PVA host for in situ reduced sample and the presence of the added silver nanoparticles in the PVA host (ex situ reduced). The optical band gaps of the films are evaluated using the UV-Vis spectrophotometer and discussed. The effect of PVA-encapsulated silver nanoparticles as a simple SPR sensor for sensing biologically important macromolecules was investigated using BSA as a model protein. The FTIR studies are utilized for the assignments of the functional groups of PVA along with the indications of the interactions of the silver nanoparticles inside the host. SEM images confirm the formation of the composites and also confirm the presence of silver particles in PVA in the case of in situ reduction. DSC thermograms are used to investigate the alignment in the melting peaks and the glass transition temperature after the addition of silver nanoparticles, irrespective of their reduction and incorporation methodology.

Acknowledgments One of the authors 'A. N' would like to thank the University Grants Commission, New Delhi for the financial assistance.

Open Access This article is distributed under the terms of the Creative Commons Attribution License which permits any use, distribution and reproduction in any medium, provided the original author(s) and source are credited.

\section{References}

Akamatsu K, Takei S, Mizuhata M, Kajinami A, Deki. S, Takeoka S, Fujii M, Havashi S, Yamamoto K (2000) Preparation and characterization of polymer thin films containing silver and silver sulfide nanoparticles. Thin Solid Films 359:55-60

Anker JN, Hall WP, Lyandres O, Shah NC, Zhao J, Van Duyne RP(2008) Biosensing with plasmonic nanosensors. Nature 7:442-453

Chou KS, Ren CY (2000) Synthesis of nanosized silver particles by chemical reduction method. Mater Chem Phys 64:241-246

Clemenson S, Alcouffe P, David L, Espuche E (2006) Structure and morphology of membranes prepared from polyvinyl alcohol and silver nitrate: influence of the annealing treatment and of the film thickness. Desalination 200:437-439
Clemenson S, Leonard D, Sage D, David L, Espuche EJ (2008) Metal nanocomposite films prepared in situ from PVA and silver nitrate. Study of the nanostructuration process and morphology as a function of the in situ routes. Polym Sci A 46:2062-2071

Devi CU, Sharma AK, Rao VVRN (2002) Electrical and optical properties of pure and silver nitrate-doped polyvinyl alcohol films. Mater Lett 56:167-174

Diez I, Pusa M, Kulmala S, Jiang H, Walther A, Goldmann AS, Muller AHE, Ikkala O, Ras RHA (2009) Color tunability and electrochemiluminescence of silver nanoclusters. Ras Angewandte Chemi 48:2122-2125

Ershov BG, Janata E, Henglein A, Fojtik A (1993) Silver atoms and clusters in aqueous solution: absorption spectra and the particle growth in the absence of stabilizing $\mathrm{Ag}+$ ions. J Phys Chem 97:4589-4594

Filippo E, Serra A, Manno D (2009) Poly (vinyl alcohol) capped silver nanoparticles as localized surface plasmon resonancebased hydrogen peroxide sensor. Sens Actuators B 138:625-630

Fusell G, Thomas J, Scanlon J, Lowman A, Marcolongo M (2005) The effect of protein-free versus protein-containing medium on the mechanical properties and uptake of ions of PVA/PVP hydrogels. J Biomater Sci Polym Ed 16:489-503

Gautam A, Ram S (2009) Preparation and thermomechanical properties of Ag-PVA nanocomposite films. Mater Chem Phys 119:266-271

Hansen UK (1981) Molecular aspects of ligand binding to serum albumin. Pharmacol Rev 33:17-53

Hopkins DS, Pekker D, Goldbart PM, Bezryadin A (2005) Quantum interference device made by DNA templating of superconducting nanowires. Science 308:1762-1765

Hussain I, Brust M, Papworth AJ, Cooper AI (2003) Preparation of acrylate-stabilized gold and silver hydrosols and gold-polymer composite films. Langumuir 19:4831-4835

Karthikeyan B (2005) Spectroscopic studies on Ag-polyvinyl alcohol nanocomposite films. Physica B 364:328-332

Khanna PK, Gokhale R, Subbarao VVVS (2004) Poly (vinyl pyrolidone) coated silver nano powder via displacement reaction. J Mater Sci 39:3773-3776

Khanna PK, Singh N, Charan S, Subbarao VVVS, Gokhale R, Mulik UP (2005) Synthesis and characterization of Ag/PVA nanocomposite by chemical reduction method. Mater Chem Phys 93:117-121

Khanna PK, More P, Jawalkar J, Patil Y, Koteswar Rao N (2009) Synthesis of hydrophilic copper nanoparticles: effect of reaction temperature. J Nanopart Res 11:793-799

Lee J, Bhattacharyya D, Easteal AJ, Metson JB (2008) Properties of nano- $\mathrm{ZnO} /$ poly (vinyl alcohol)/poly(ethylene oxide) composite thin films. Curr Appl Phys 8:42-47

Mbhele ZH, Salemane MG, Van Sittert CGCE, Nedeljkovic JM, Djokovic V, Luyt AS (2003) Fabrication and characterization of silver-polyvinyl alcohol nanocomposites. Chem Mater 15:5019-5024

McDonagh C, Burke CS, MacCraith BD (2008) Optical chemical sensors. Chem Rev 108:400-422

Mock JJ, Hill RT, Degiron A, Zauscher S, Chilkoti A, Smith DR (2008) Distance-dependent plasmon resonant coupling between a gold nanoparticle and gold film. Nano Lett 8:2245-2252

Monti OLA, Fourkas JT, Nesbitt DJ (2004) Diffraction-limited photogeneration and characterization of silver nanoparticles. J Phys Chem B 108:1604-1612

Mulfinger L, Solomon SD, Bahadory M, Jeyarajasingam AV, Rutkowsky SA, Boritz C (2007) Synthesis and study of silver nanoparticles. J Chem Educ 84:322

Nesher G, Marom G, Anvir D (2008) Metal-polymer composites: synthesis and characterization of polyaniline and other polymer at silver compositions. Chem Mater 20:4425-4432 
Porel S, Singh S, Sree Harsha S, Narayana Rao D, Radhakrishnan TP (2005) Nanoparticle-embedded polymer: in situ synthesis, freestanding films with highly monodisperse silver nanoparticles and optical limiting. Chem Mater 17:9-12

Rozenberg BA, Tenne R (2008) Polymer-assisted fabrication of nanoparticles and nanocomposites. Progress in polymer science 33:40-112

Temgire MK, Joshi SS (2004) Optical and structural studies of silver nanoparticles. Radiat Phys Chem 71:1039-1044

Valanciunaite J, Bagdonas S, Streckyte G, Rotomskis R (2006) Spectroscopic study of TPPS4 nanostructures in the presence of bovine serum albumin. Photochem Photobiol Sci 5:381-388

Wang P-H, Wu Y-Z, Zhu Q-R (2002) Polymer metal composite particles: polymer core and metal shell. J Mater Sci Lett 21:1825-1828

Yu D-G, Lin W-C, Lin C-H, Chang L-M, Yang M-C (2007) An in situ reduction method for preparing silver/poly(vinyl alcohol) nanocomposite as surface-enhanced Raman scattering (SERS)-active substrates. Mater Chem Phys 101:93-98
Zavyalov SA, Pivkina AN, Schoonman J (2002) Formation and characterization of metal-polymer nanostructured composites. Solid State Ionics 147:415-419

Zeng R, Rong MZ, Zhang MQ, Liang HC, Zeng HM (2002) Laser ablation of polymer-based silver. Appl Surf Sci 187:239-247

Zhang Z, Zhao B, Hu L (1996) PVP protective mechanism of ultrafine silver powder synthesized by chemical reduction processes. J Solid State Chem 121:105-110

Zheng M, Gu M, Jin Y, Jin G (2000) Optical properties of silverdispersed PVP thin film. Mater Res Bull 36:853-859

Zhong Z, Wang D, Cui Y, Bockrath MW, Lieber CM (2003) Nanowire crossbar arrays as address decoders for integrated nanosystems. Science 302:1377-1379

Zhou Y, Yu SH, Wang CY, Li XG, Yu CZ (1999) A novel ultraviolet irradiation photoreduction technique for the preparation of single-crystal $\mathrm{Ag}$ nanorods and $\mathrm{Ag}$ dendrites. Adv Mater $11: 850-852$ 